POS PROCEEDINGS

\title{
BurstCube: Concept, Performance, and Status
}

\author{
Jacob R. Smith* \\ NASA/GSFC/CRESST \\ E-mail: jacob.r.smith@nasa.gov
}

Michael S. Briggs ${ }^{a}$, Alessandro Bruno $^{b}$, Eric Burns $^{b}$, Regina Caputo ${ }^{b}$, Brad Cenko $^{b}$, Antonino Cucchiara ${ }^{c}$, Georgia de Nolfo ${ }^{b}$, Sean Griffin ${ }^{d}$, Lorraine Hanlon ${ }^{e}$, Dieter H. Hartmann $^{f}$, Michelle Hui ${ }^{g}$, Alyson Joens ${ }^{h}$, Carolyn Kierans ${ }^{b}$, Dan Kocevski ${ }^{g}$, John Krizmanic $^{d}$, Amy Lien ${ }^{d}$, Sheila McBreen ${ }^{e}$, Julie E. McEnery ${ }^{b}$, Lee Mitchell ${ }^{i}$, David Morris $^{c}$, David Murphy ${ }^{e}$, Jeremy S. Perkins ${ }^{b}$, Judy Racusin $^{b}$, Peter Shawhan $^{j}$, Teresa Tatoli $^{d}$, Alexey Uliyanov ${ }^{e}$, Sarah Walsh ${ }^{e}$, Colleen Wilson-Hodge ${ }^{g}$

${ }^{a}$ University of Alabama, Huntsville

${ }^{b} N A S A / G S F C$

${ }^{c}$ University of the Virgin Islands

${ }^{d}$ NASA/GSFC/CRESST

${ }^{e}$ University College Dublin

${ }^{f}$ Clemson University

${ }^{g}$ NASA/MSFC

${ }^{h}$ George Washington University

${ }^{i}$ Naval Research Laboratory

${ }^{j}$ University of Maryland, College Park

The first simultaneous detection of a short gamma-ray burst (SGRB) with a gravitational-wave (GW) signal ushered in a new era of multi-messenger astronomy. In order to increase the number of SGRB-GW simultaneous detections, we need full sky coverage in the gamma-ray regime. BurstCube, a CubeSat for Gravitational Wave Counterparts, aims to expand sky coverage in order to detect and localize gamma-ray bursts (GRBs). BurstCube will be comprised of 4 Cesium Iodide scintillators coupled to arrays of Silicon photo-multipliers on a 6U CubeSat bus (a single $\mathrm{U}$ corresponds to cubic unit $\sim 10 \mathrm{~cm} \times 10 \mathrm{~cm} \times 10 \mathrm{~cm}$ ) and will be sensitive to gamma-rays between $50 \mathrm{keV}$ and $1 \mathrm{MeV}$, the ideal energy range for GRB prompt emission. BurstCube will assist current observatories, such as Swift and Fermi, in the detection of GRBs as well as provide astronomical context to gravitational wave events detected by Advanced LIGO, Advanced Virgo, and KAGRA. BurstCube is currently in its development and testing phase to prepare for launch readiness in the fall of 2021 . We present the mission concept, preliminary performance, and status.

36th International Cosmic Ray Conference -ICRC2019-

July 24th - August 1st, 2019

Madison, WI, U.S.A.

${ }^{*}$ Speaker. 


\section{Introduction}

BurstCube detections of short gamma-ray burst (SGRBs) in the multimessenger era will provide critical observations needed to unveil the origins and properties of binary neutron star (BNS) mergers, and possibly neutron star black hole (NSBH) mergers, if discovered. Jets that power GRBs are ultra-relativistic, highly-collimated beamed outflows and are some of the shortest-lived and most energetic jets in the universe [1]. Electromagnetic follow-up observations of these jet sources revealed their cosmological origins, showed that they have extremely compact central engines and constitute two classes based on emission duration. SGRBs have emission durations less than 2 seconds and long duration burst (LGRBs) last longer than 2 seconds [2]. The merger hypothesis for SGRBs [3] was developed from these observations and spectacularly confirmed with GW170817/GRB17017A [4, 5, 6].

GW observations directly probe the central engine, and combining this with information from the electromagetic sector offers additional insights into these events. Coincident detections enable additional observations from a wealth of multi-wavelength EM telescopes in operation today and the early 2020s [7]. BurstCube observations will provide complementary information to other GRB monitors. From these we will learn about the energetics of the jet, bulk Lorentz factor of the outflow and geometry of the emission region. Time delay between the merger and the SGRB allows us to study jet physics, neutron star equation of state, and the speed of gravity [8]. Fundamental progress can be achieved for NS mergers and GRB jet physics with the combined observations of the GW network, current GRB monitors, and BurstCube.

\section{BurstCube}

Thanks to the growth and development of small-sat and CubeSat technologies it is now achievable to build a CubeSat to detect the bright signals from GRBs. These proceedings detail a $6 \mathrm{U}$ CubeSat (a single $U$ corresponds to cubic unit $\sim 10 \mathrm{~cm} \times 10 \mathrm{~cm} \times 10 \mathrm{~cm}$ ) [9] mission called BurstCube that is based on the Dellingr platform developed at GSFC [10]. The main limiting factors in developing a mission like BurstCube are mass, power, and volume but the advent of lowpower, low-volume SiPMs enables a workable detector readout design. We describe the BurstCube CubeSat, which is designed to provide rapidly available high-resolution temporal, spectral, and localization data. Even rough localizations will be helpful to verify weak GW signals, as the temporal coincidence is the most important measurement alerting the community to a potential on-axis event. BurstCube increases the sky coverage of existing facilities, and is optimized for detecting SGRBs, all for a small fraction of the cost of a larger mission.

BurstCube is a $6 \mathrm{U}$ CubeSat divided into a $4 \mathrm{U}$ instrument package, and $2 \mathrm{U}$ of spacecraft subsystems (Fig. 1). The format of the $6 \mathrm{U}$ spacecraft drives the instrument mass, power, and volume budget. The instrument design is similar to Fermi-GBM [11], except BurstCube uses Thallium doped Cesium Iodide $(\mathrm{CsI}(\mathrm{Tl})$ ) crystal as the detection medium covering the energy range from $50 \mathrm{keV}-1 \mathrm{MeV}$ with high efficiency and adequate energy resolution. These crystals are inexpensive and have a long track record of use in $\gamma$-ray astronomy. A design philosophy is to rely on proven well-tested technology. The dimensions of the CsI employed for BurstCube are $9 \mathrm{~cm}$ in diameter and $1.9 \mathrm{~cm}$ thick, with the maximum dimension dictated by the size of the $6 \mathrm{U}$ CubeSat. 


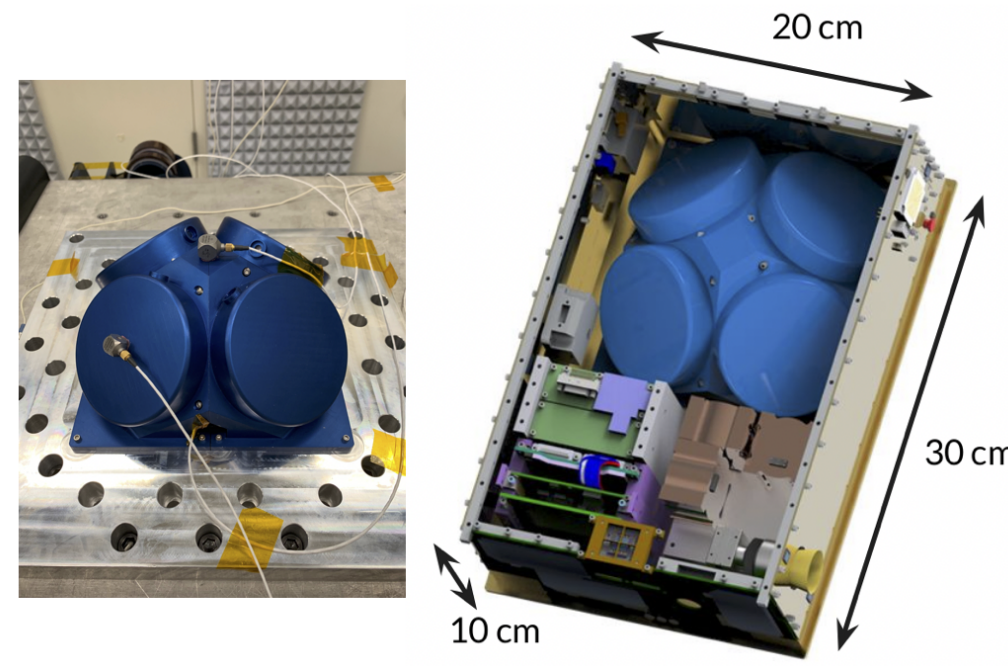

Figure 1: Internal view of the BurstCube instrument and spacecraft components (right), and the instrument mounted on a vibration (vibe) table (left). For the vibe test, one quarter was instrumented with a CsI(Tl) crystal and a proto-flight SiPM array front-end electronics board and the other three with mock masses. Tests consisted of flight-like vibe amplitudes on all three axes. BurstCube vibe was completed and successfully passed in June 2019.

Readout of each crystal is done by an array of 116 low-power and low-voltage (56-60 V) Hammamatsu Silicon Photomultipliers (SiPMs). Compared to the more conventional photomultiplier tubes, SiPMs significantly reduce mass, volume, power, and cost. However, even in this compact design, the detector presented here is competitive with the state of the art system (Fermi-GBM). A single BurstCube crystal has $70 \%$ of the effective area of a single GBM crystal at $100 \mathrm{keV}$; see Fig. 3. However, GBM has better sky-uniformity and better localizations. The $4 \mathrm{U}$ instrument package consists of four Single Quarter Detectors (SQD) each with a PTFE wrapped crystal housed inside an $\mathrm{Al}$ structure with quartz glass and silicone optical padding between the crystal and a SiPM frontend electronics (FEE) board, see Fig. 2. The combination of scintillation crystals and new readout devices makes it possible to consider a compact, low-power instrument that is readily deployable on a CubeSat platform.

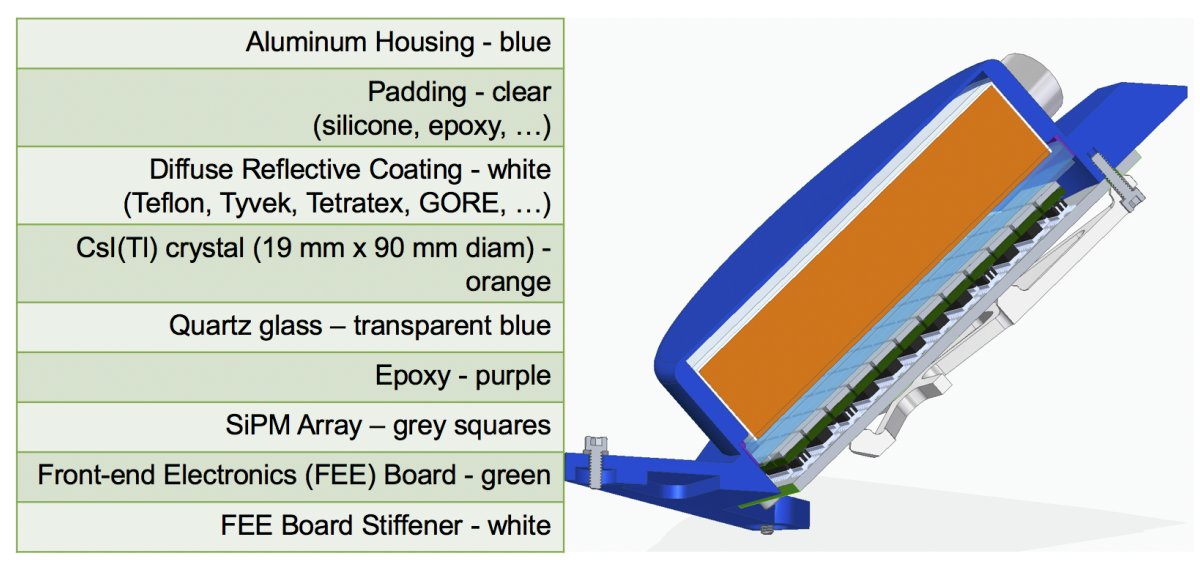

Figure 2: Cross-section view of the BurstCube SQD (right) with a description of the internal components (left).

To estimate the BurstCube effective area and resulting sensitivity, we simulated a single CsI detector using MEGAlib [12] (which provides an interface to GEANT4). The simulated BurstCube effective area is complementary to GBM (Fig. 3), despite the smaller detectors. BurstCube will lo- 


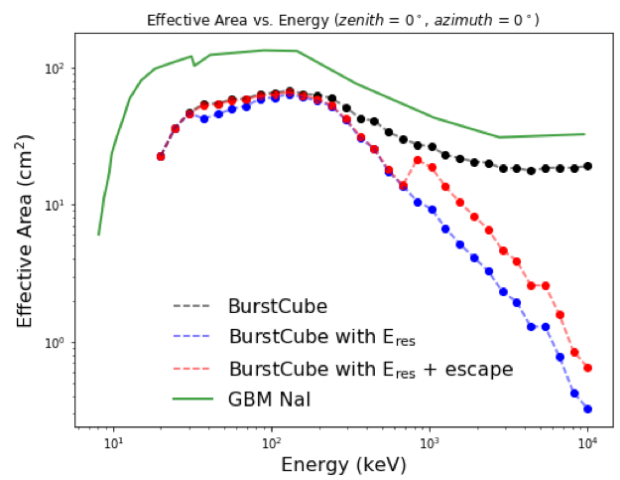

Figure 3: Despite the constraints of a CubeSat, detector simulations show that BurstCube achieves an effective area of $70 \%$ of GBM at $100 \mathrm{keV}$ and $15^{\circ}$ incidence The effective area as a function of energy, and the corresponding curve for the larger GBM NaI detectors are shown for reference.

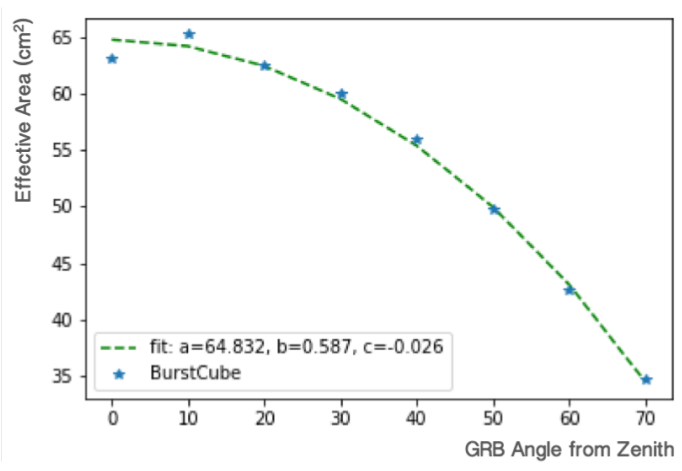

Figure 4: The cylindrical BurstCube detectors make maximum use of space and still achieve an effective area cosine dependence on incidence angle expected for a thin detector which is needed for GRB localizations.

calize GRBs in a similar manner to BATSE or GBM $[13,14]$. The approximate cosine dependence $\left(\sim \cos (\theta)^{0.59}\right)$ of the effective area with incidence angle (Fig. 4$)$ is leveraged to localize GRBs by measuring the relative brightness of the burst between the four detectors. The detector count rates will be matched to a previously computed table of relative detector responses as a function of spacecraft coordinates. For $90 \%$ of SGRBs we are expecting localizations better than $30^{\circ}$ radius.

The BurstCube instrument is housed in a CubeSat bus based on the Dellingr platform, a modular platform designed to be easily modifiable for a variety of $6 \mathrm{U}$ CubeSat architectures (see Fig. 1). It includes 3 axis-stabilized pointing control, star tracker, deployable mounted solar panels, and is designed for the Planetary Systems Corporation (PSC) 6U canister standard ${ }^{1}$ [15]. A CubeSat implementation of the Vulcan communications system is currently being developed for use in BurstCube. The Vulcan radio will enable rapid GRB location distribution through the Tracking and Data Relay Satellite System (TDRSS).

\section{Performance}

In addition to detector simulations, expected performance of the BurstCube mission has been determined from laboratory measurements using proto-flight components of the instrument. In the last year we have constructed a proto-flight of the instrument that consists of a front-end electronics (FEE) board and a SQD. The FEE board is where the array of 116 SiPMs and associated summing electronics is installed. We have characterized the temperature response, noise behavior, and breakdown voltage of the SiPM array with the use of various gamma-ray radiation sources in the energy range from $26 \mathrm{keV}$ up to $1.33 \mathrm{MeV}$. Most of the characterization of the BurstCube SiPM FEE board has been done using a $10 \mathrm{~cm}$ diameter, $2 \mathrm{~cm}$ thick $\mathrm{CsI}(\mathrm{Na})$ test crystal. Fig. 5 shows our measured noise response and I-V curve, representing the breakdown voltage, of the SiPM FEE as a function of bias voltage and temperature. Not shown here is the energy response to the $26 \mathrm{keV}$ and $59 \mathrm{keV}$

\footnotetext{
${ }^{1}$ Also compatible with the NanoRacks $6 \mathrm{U}$ dispenser.
} 
gamma-ray emission from an Americium-241 radiation source. The $26 \mathrm{keV}$ photo-peak is distinguishable from noise below $38 \mathrm{C}$ and the $59 \mathrm{keV}$ photo-peak is distinguishable from noise below $41 \mathrm{C}$. Based on initial orbital temperature analysis, the hottest expected temperature of BurstCube is below $35 \mathrm{C}$. These results indicate that BurstCube will detect gamma-rays below its low energy goal of $50 \mathrm{keV}$.
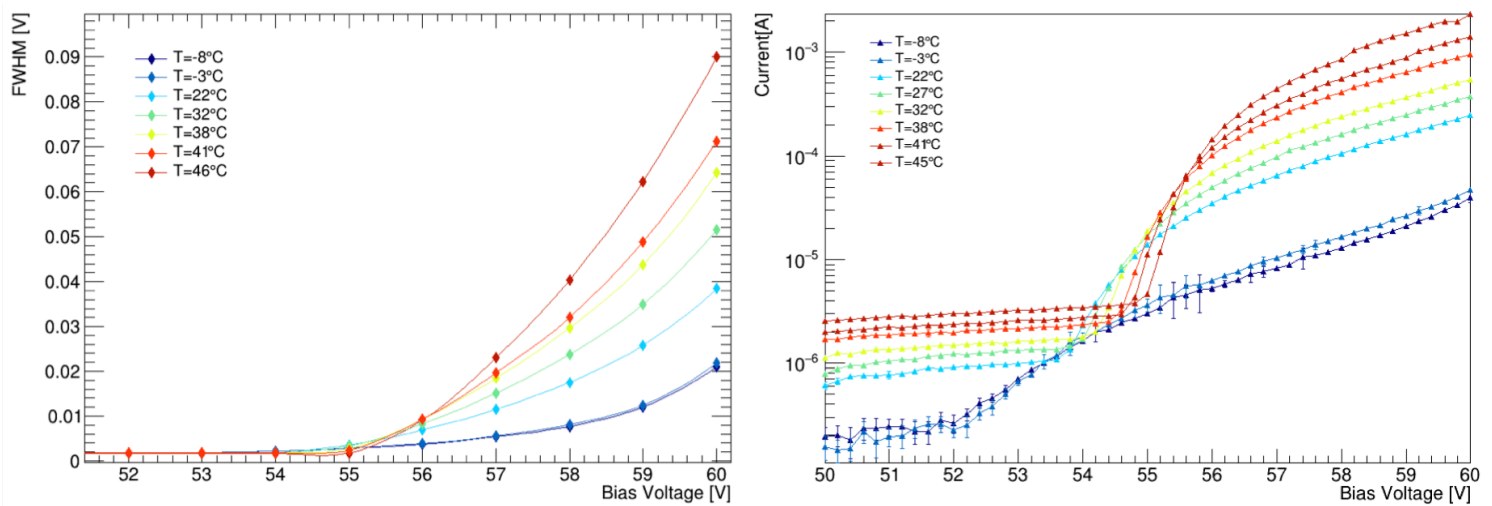

Figure 5: Noise of the SiPM array (left), represented as FWHM [V] as a function of bias voltage, and breakdown voltage (right), represented in terms of an I-V curve as a function of bias voltage, was measured with the BurstCube proto-flight SiPM FEE board. BurstCube can detect down to $26 \mathrm{keV}$ and achieve constant gain through temperature compensation within its expected flight temperature environment.

The breakdown voltage of SiPMs have a strong dependence on temperature. During the flight operations, BurstCube will experience a range of temperatures between $5-35^{\circ} \mathrm{C}$. As a result we need to demonstrate an ability to compensate for these fluctuations in temperature. Shown in right panel of Fig. 5 are lab measurements of the I-V curve over a range of temperatures. The breakdown voltage at each temperature is indicated by the sudden increase of current at a particular bias voltage. An on-board look-up-table (LUT) will be used in flight to compensate the temperature variations by adjusting the applied bias voltage. In this way, BurstCube can maintain constant gain within the temperature fluctations of the mission lifetime.

Data have been collected with the BurstCube SiPM FEE board with radiation sources including Am-241, Bi-207, Cs-137 and Co-60 to measure the energy response from $26 \mathrm{keV}$ up to 1.33 $\mathrm{MeV}$ with a test crystal at room temperature, see Fig. 6. BurstCube shows a linear response and sufficient energy resolution over the desired energy range of the mission. Given the measured breakdown voltage over the expected temperature range we expect to have constant linear gain and good resolution with the BurstCube 116 SiPM array.

\section{Current Status}

BurstCube is currently approaching the Critical Design Review (CDR) for the instrument detectors and readout and the Systems Peer Review (SPR) for the spacecraft bus components. The Critical Design Review for BurstCube will verify the final design to initiate the flight instrument build. The spacecraft SPR will serve as the decision point to finalize the components and interfaces within the spacecraft bus and components. Key milestones that have been completed leading up 

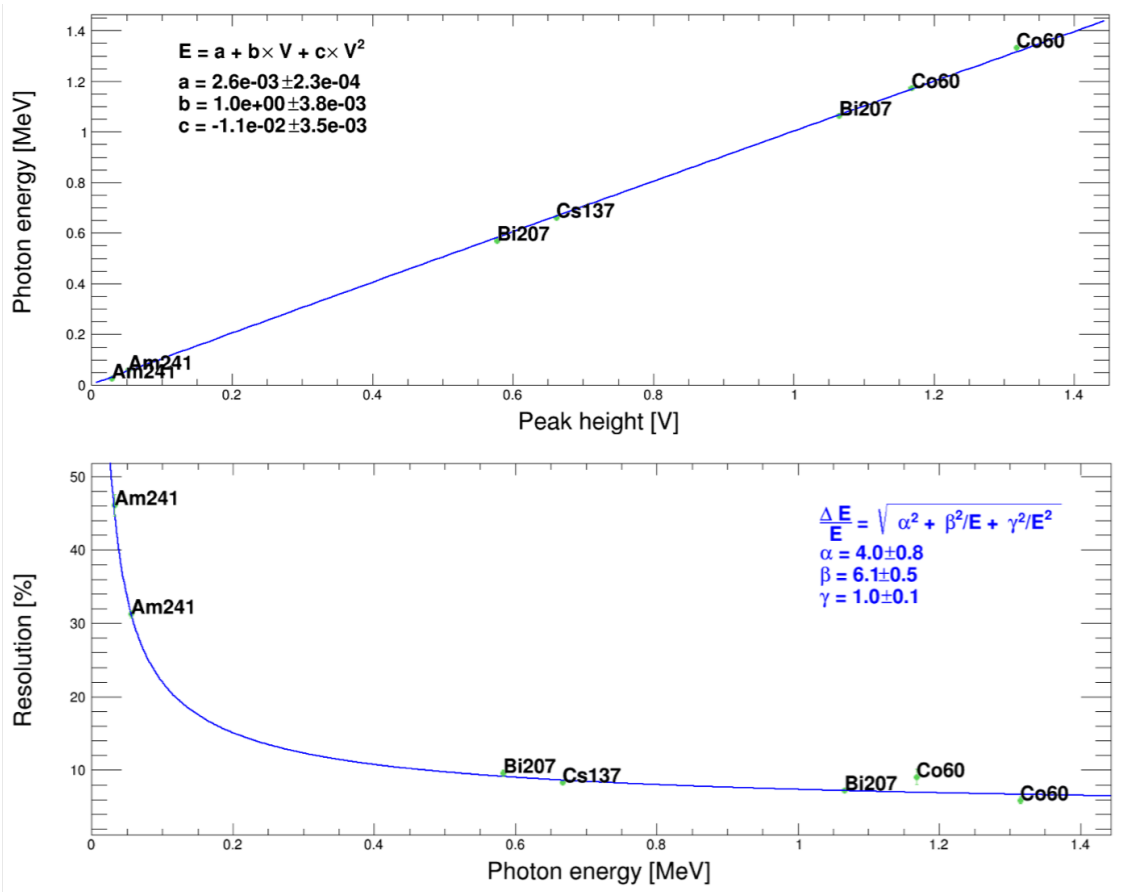

Figure 6: Energy linearity (top) and resolution (bottom) for the BurstCube SiPM FEE with the test crystal using gamma-ray radiation sources between $26 \mathrm{keV}$ up to $1.33 \mathrm{MeV}$. The SiPM array is linear and has sufficient energy resolution over the desired energy range of the mission.

to the instrument CDR include: design, integration and test of the $\mathbf{1 1 6}$ array SiPM front-end electronics (FEE) board; design of the instrument power supply; construction of a protoflight SQD; completion of the proto-flight environmental qualification vibration and thermal vacuum tests.

Environmental qualification of the SQD validates the BurstCube flight instrument design. Following General Environmental Verification Standard (GEVS) for Goddard Space Flight Center (GSFC) flight programs and projects [16], in July 2019, we have demonstrated by test that BurstCube will satisactorily perform in its mission environment. GEVS provides the requirements test levels for vibration and thermal vacuum tests of flight or flight-like hardware. The energy response of BurstCube to gamma-ray radiation source was used to demonstrate BurstCube's performance before, during and after environmental tests. Constant gain, linear energy response, and energy resolution was maintained after vibration tests and during thermal vacuum cycles. During the temperature cycles of thermal vacuum tests we were able to maintain constant electronics gain and energy response by compensating the bias voltage of the 116 array SiPM FEE board. Following an independent assessment on the technology readiness level (TRL), the BurstCube instrument will be at TRL 6 .

Between the successful completion of the environmental tests and CDR, we plan to: build a flat-sat of the instrument digital electronics with an FPGA and flight-like bias power supply; test FPGA code with instrument and spacecraft interfaces; test FPGA trigger, data processing, and temperature compensation codes; finalize the requirements of the instrument flight software; finalize requirements for the ground pipelines and science analysis software; and complete the full 
instrument detector simulations. Calibration test plans are also currently in the draft stage that will be used for the flight build of BurstCube.

\section{References}

[1] J. Goodman, Are gamma-ray bursts optically thick?, ApJ 308 (Sept., 1986) L47-L50.

[2] C. Kouveliotou, C. A. Meegan, G. J. Fishman, N. P. Bhat, M. S. Briggs, T. M. Koshut et al., Identification of two classes of gamma-ray bursts, ApJ 413 (Aug., 1993) L101-L104.

[3] R. Narayan, B. Paczynski and T. Piran, Gamma-ray bursts as the death throes of massive binary stars, ApJ 395 (Aug., 1992) L83-L86, [astro-ph/920 4001 ].

[4] Ligo Scientific Collaboration and Virgo Collaboration collaboration, B. P. Abbott, R. Abbott, T. D. Abbott, F. Acernese, K. Ackley, C. Adams et al., Gw170817: Observation of gravitational waves from a binary neutron star inspiral, Phys. Rev. Lett. 119 (Oct, 2017) 161101.

[5] A. Goldstein, P. Veres, E. Burns, M. S. Briggs, R. Hamburg, D. Kocevski et al., An ordinary short gamma-ray burst with extraordinary implications: Fermi-GBM detection of GRB 170817a, The Astrophysical Journal 848 (oct, 2017) L14.

[6] V. Savchenko, C. Ferrigno, E. Kuulkers, A. Bazzano, E. Bozzo, S. Brandt et al., INTEGRAL detection of the first prompt gamma-ray signal coincident with the gravitational-wave event GW170817, The Astrophysical Journal 848 (oct, 2017) L15.

[7] V. A. Villar, J. Guillochon, E. Berger, B. D. Metzger, P. S. Cowperthwaite, M. Nicholl et al., The combined ultraviolet, optical, and near-infrared light curves of the kilonova associated with the binary neutron star merger GW170817: Unified data set, analytic models, and physical implications, The Astrophysical Journal 851 (dec, 2017) L21.

[8] B. P. Abbott, R. Abbott, T. D. Abbott, F. Acernese, K. Ackley, C. Adams et al., Gravitational waves and gamma-rays from a binary neutron star merger: GW170817 and GRB 170817a, The Astrophysical Journal 848 (oct, 2017) L13.

[9] "Cubesat design specification rev. 13.” The CubeSat Program, Cal Poly SLO.

[10] M. Johnson, T. Bonalsky, D. Chornay, C. Clagett, A. Cudmore, A. Ericsson et al., Dellingr- A Path to Compelling Science with CubeSats, in European Planetary Science Congress, pp. EPSC2015-720, Oct, 2015.

[11] C. Meegan et al.ApJ 702 (Sept., 2009) 791-804, [0 908 . 0450 ].

[12] A. Zoglauer, R. Andritschke and F. Schopper, "megalib the medium energy gamma-ray astronomy library", .

[13] G. J. Fishman et al., Overview of Observations from BATSE on the Compton Observatory, A\&AS 97 (Jan., 1993) 17.

[14] V. Connaughton et al., Localization of Gamma-Ray Bursts Using the Fermi Gamma-Ray Burst Monitor, ApJS 216 (Feb., 2015) 32, [1411.2685].

[15] “Canisterized satellite dispenser data sheet." 2002337F.

[16] "General environmental verification standard (gevs) for gsfc flight programs and projects." GSFC-STD-7000A, 2013. 